\title{
Emergence, Dependence, and Fundamentality
}

\author{
Olley Pearson ${ }^{1}$
}

Received: 17 December 2015/ Accepted: 24 March 2017/Published online: 8 April 2017

(C) The Author(s) 2017. This article is an open access publication

\begin{abstract}
In a recent paper Barnes proposes to characterize ontological emergence by identifying the emergent entities with those entities which are both fundamental and dependent. Barnes offers characterizations of the notions of fundamentality and dependence, but is cautious about committing to the specifics of these notions. This paper argues that Barnes's characterization of emergence is problematic in several ways. Firstly, emergence is a relation, and merely delimiting relata of this relation tells us little about it. Secondly, the group of entities delimited as dependent and fundamental do not appear to be the group of emergent entities. Rather, some entities appear to be dependent and fundamental and not emergent, whilst other entities appear to be emergent and not dependent and fundamental. The moral drawn is that in order to provide a characterization of emergence one must go beyond what Barnes says explicitly. It is also shown that a potentially fruitful way of doing this would be to further specify the notion of dependence at issue revealing it to be asymmetric and perhaps merely nomological.
\end{abstract}

In a recent paper Barnes offers a characterization of emergence (2012). Emergent entities are said to be those which are both fundamental and dependent. In giving this characterization Barnes utilizes some meta-ontological views that will not be universally accepted. I will not challenge these views themselves but instead the use Barnes makes of them. In Sect. 1, I provide a brief description of emergence and then a proposed characterization of it offered by Barnes, which I call FundDep. In Sect. 2, I argue that to get the most out of FundDep one must read more into it than is explicitly stated. FundDep only explicitly delimits the class of putatively emergent entities, but emergence is a relation and delimiting the relata of a relation

Olley Pearson

f.o.c.pearson@durham.ac.uk

1 Department of Philosophy, Durham University, 50 Old Elvet, Durham DH1 3HN, UK 
is not sufficient to characterize it. In Sects. 3 and 4, I argue that the group of entities delimited by FundDep is not the group of emergent entities according to common conceptions of that notion. There are plausibly emergent entities that lie outside the proposed group (Sect. 3) and entities within the proposed group which are not emergent (Sect. 4). These faults generally follow from the notion of dependence central to FundDep, which is hence shown to be inadequate. However, Barnes does not fully commit to this notion, and so in Sect. 5 I consider how one might build on what Barnes says by developing a more nuanced characterization of dependence.

\section{Barnes's Proposed Characterization of Emergence}

Talk of emergence has arisen in a number of different fields, including sociology, biology, physics, and philosophy, and it has the potential to further clarify topics within these fields. ${ }^{1}$ For example, taking the mind to be emergent might help accommodate certain intuitions about physicalism and non-reduction. However, to date advances have been hindered through a lack of clarity surrounding the notion of emergence itself. This is a failing that Barnes is trying to help correct.

Barnes is concerned with ontological emergence, which roughly speaking is the emergence of new entities (where entities include both objects and properties). ${ }^{2}$ For example, it might be thought to occur if a collection of particles has causal powers distinct from the mere combination of the powers of the individual particles. ${ }^{3}$ Ontological emergence will also be the subject of this paper.

Barnes proposes to characterize emergence by identifying the emergent entities with those entities which are both dependent and fundamental. However, as Barnes points out, this is not in itself novel insofar as previous authors have described emergent entities as dependent and fundamental. Prior use of the notion of fundamentality has not always been clear or unanimous. One apparent example of it is to describe emergent properties as fundamental in order to point out that while these are properties of so called higher-level sciences, such as psychology, they may nevertheless have influence at the fundamental level, which is commonly taken to be the level of physics. ${ }^{4}$ The use of the notion of dependence has also been varied, but the general aim appears to be to pick out that the emergent entities are in some way reliant on non-emergent entities. ${ }^{5}$ In O'Connor (1994) emergent entities are described as both fundamental and dependent; more recently, in Wilson

\footnotetext{
${ }^{1}$ Cf. Sawyer (2001), Rothschild (2006), Laughlin (1999), and Van Gulick (2001), respectively.

2 For discussion of the distinction between ontological and epistemological emergence see Van Gulick (2001). Chalmers (2006) makes a related distinction between strong and weak emergence. Wilson (2015) (see also her 1999 and 2011) distinguishes between weak and strong forms of ontological emergence; in Wilson's terms, the notion of emergence Barnes aims to characterize is of the strong rather than weak variety.

3 Cf. Barnes (2012: 890), Maudlin (2007: ch. 2), and Wilson (1999, 2005, 2015).

${ }^{4}$ Cf. the use made in McLaughlin (1997), Chalmers (2006), and Wilson (1999, 2005, 2015).

5 Cf. van Cleve (1990) and Kim (2006).
} 
(2013, 2015) (a very similar early draft of which Barnes cites) emergence is characterized in these terms. ${ }^{6}$

What is new in Barnes's discussion of emergence is the particular interpretation of dependence and fundamentality that Barnes intends. Barnes says that we can characterize the fundamental entities by identifying them with the truth-makers for existence claims, or the entities that God must create in order to make the world as it is. So for Barnes something either is or is not derivative; derivativeness cannot come in degrees. ${ }^{7}$ For example, it is true that my pencil exists. However, according to the view Barnes is proposing this is not made true by the pencil itself but instead by the elementary particles that make up the pencil. All God needs to do to make a world containing the pencil is to make the particles (arranged in a certain way). So the pencil is derivative, while the particles composing it are fundamental.

For Barnes the characterization of dependence is quite separate from the characterization of fundamentality, and she offers the following:

An entity $x$ is dependent iff for all possible worlds $w$ and times $t$ at which a duplicate of $x$ exists, that duplicate is accompanied by other concrete, contingent objects in $w$ at $t$. (2012: 880). ${ }^{8}$

This notion of dependence captures what might be intuitively thought when one considers that a table, for example, is dependent upon its parts because the table cannot exist unless it has some parts that exist at the same time.

Fundamentality and dependence are thus characterized independently. (Henceforth I will capitalise these expressions to be clear these interpretations are intended.) Furthermore, Barnes suggests they mark distinctions which needn't coincide. This gives us four ontological categories: the Fundamental Independent entities, the Fundamental Dependent entities, the Derivative Independent entities, and the Derivative Dependent entities.

Barnes is in fact hesitant to commit to these characterizations of Fundamentality and Dependence as analyses, because she believes that the notions are primitive. ${ }^{9}$ However, they do offer a clear proposal for characterizing emergence, which I will consider in the course of this paper. According to this view, referred to as 'FundDep', the emergent entities are the Fundamental Dependent entities. That is, an emergent entity will be a truth-maker for an existence claim or something God must create to make the world the way it is. It will also be an entity which is at all times accompanied by other concrete, contingent entities, and whose otherworldly duplicates are all, at all times, accompanied by other concrete, contingent entities.

\footnotetext{
6 There are also authors such as Kim (2006) who talk of emergent entities being dependent and making fundamental differences.

7 Cf. Cameron (2008).

${ }^{8}$ Barnes speaks of 'objects', I am interpreting this loosely to allow property instances to be objects. If Barnes would not allow this, her account is obviously inadequate as there might be emergent properties in a trope ontology (as Barnes notes). Either way, the arguments of this paper stand with a change of examples.

9 Barnes also accepts that her characterization of Dependence might be inadequate for necessarily existing entities. However, this paper focuses on contingent entities throughout.
} 
FundDep shows initial promise, as it provides a clear interpretation of the notion of emergence through interpreting the notions of fundamentality and dependence. Furthermore, it does so in a way that captures crucial dimensions of emergence. The notion of Fundamentality specifies that emergence is an ontological issue (not merely an epistemological one), while Dependence makes clear we are concerned with a particular subset of entities which are in some way related to other entities. In the following sections, however, I will raise some concerns with FundDep.

\section{The Emergence Relation is Not Captured by FundDep}

The idea that emergence is a relation between an emergent entity and the base it emerges from goes back to the notion's birth with the British Emergentists and their identification of emergence with nomological relations, such as are captured by Mill (1843/1919)'s heteropathic and Broad (1925)'s trans-ordinal laws. Attempts to characterize this relation have continued within discussions of emergence, as can be seen in the more recent work of, for example, Van Gulick (2001), Kim (2006), Chalmers (2006), and Wilson (2015).

That emergence is a relation is also evident in potential cases of emergence. For example, persons might be thought to be emergent from their bodies. ${ }^{10}$ Nick's body is a collection of physical particles, but Nick is distinct from this as he has characteristics such as consciousness that the physical parts, even in combination, lack. ${ }^{11}$ Nevertheless, while Nick is distinct from his body, just as Clare is distinct from hers, Nick is emergent from his body and not emergent from Clare's, and vice versa.

The point to recognize from these cases-whether or not they turn out to be genuine examples of emergence ${ }^{12}$ - is that entities are seen as emergent precisely in being seen as standing in a certain relation to the specific bases from which they emerge. If I want to know about emergence, I want to know about something that holds between Nick and his body, and not between Nick and Clare's body. In order for FundDep to characterize emergence, and to improve on other characterizations, it must therefore give an account of this relation and/or the distinctions it marks. However, no such account is explicit in FundDep. Merely delimiting the emergent entities equips us to recognize whether or not a given entity is emergent but not to recognize what an emergent entity is emergent from.

Suppose that Nick and Clare both exist in this world, as do their bodies BodyNick and BodyClare respectively. Let us suppose that Nick and Clare are each Fundamental and Dependent so that they meet FundDep's characterization of emergent entities. This does not tell us if Nick emerges from BodyNick or from BodyClare, and as such it fails to tell us something important-indeed, crucialabout emergence.

\footnotetext{
${ }^{10}$ Cf. Barnes (2012: 887), and Lowe (2008).

11 Cf. O'Connor (2000) for the idea that consciousness is emergent.

12 I do not assume that there are any genuine examples of emergence. All talk of emergent entities within this paper should be interpreted as talk of potentially emergent entities.
} 
At this point one might try to look to FundDep's notion of Dependence for help, as dependence, like emergence, is a relation. Furthermore, Wilson (2015), in surveying and systematizing possible characterizations of emergence, argues that on all characterizations it involves two elements: an emergent entity must be novel, and it must stand in a relation of dependence to its base. Dependence thus appears to be a natural place to look to for relational aspects of emergence and to address the problem I have just revealed.

Nevertheless, the way in which FundDep characterizes Dependence offers little help with the current problem. The characterization at issue delimits the Dependent entities but does not enable us to explicitly connect dependent entities to specific dependence base entities. Something is said to be Dependent if it is accompanied by something else concrete and contingent in every possible world where it or a duplicate of it exists, but this says nothing about what an entity is dependent upon. We have a characterization that enables us to sort entities into the kind related-by-adependence-relation, but we do not have a characterization that enables us to identify for any particular instance of the dependence relation what its relata are.

The problems here are general in form. Knowing the relata of a kind of relation tells one little about the relation, as is clear from the fact that two different kinds of relations may hold between all the same relata. Further, one might know that a specific group of entities are all relata of instances of a particular relational kind, without knowing for any particular instance of that relational kind what its relata are.

Returning to the case of Nick, BodyNick, Clare, and BodyClare, if we limit ourselves to what is explicit in FundDep, we are not equipped to determine that Nick is Dependent upon BodyNick and not upon BodyClare, or hence that Nick is emergent from BodyNick and not BodyClare. However, dependence is commonly understood to be a relation and so plausibly it can be further characterized in a way that enables us to avoid the problems just outlined. Crucially, a more developed characterization of dependence may determine where an instance of the dependence relation holds, perhaps determining that Nick is dependent upon BodyNick and not BodyClare, and is hence emergent from BodyNick and not BodyClare. The conclusion I draw at this stage is thus that FundDep is not a sufficient characterization of emergence. However, this is not to say that FundDep is mistaken, since it may be extended by a further characterization of the Dependence relation; it is of note, however, that such an extension is required. I will return to this issue in Sect. 5.

\section{Emergent but Not Fundamental and Dependent}

While FundDep fails to adequately characterize the relation of Dependence and hence of emergence, it does try to delimit the relata of these relations and thereby the emergent entities. However, in this section I will argue that the group of entities delimited by FundDep is not the group of emergent entities, as there are candidate emergent entities that are not Fundamental and Dependent. 
According to numerous characterizations of emergence it is a nomologically necessary relation. This idea again goes back to the founders of modern discussions of emergence. For example, it is found in Mill (1843/1919)'s use of nomological heteropathic laws, and Broad (1925)'s use of nomological trans-ordinal laws, to identify relations of emergence. The idea also continues in more contemporary discussions, where it has been argued that to be a case of emergence the relations at issue must be merely nomological. ${ }^{13}$ If the relation between an emergent entity and its base is not metaphysically necessary, it cannot be a case of identity. Given reduction is commonly taken to involve identity, this failure of metaphysical necessity thus prevents reduction and thereby encourages the recognition of the emergent entity as novel and emergent. Chalmers (2006) takes consciousness to be emergent from the physical because it is related to the physical by the laws of science but not through metaphysical necessity. Without necessarily endorsing the emergence of consciousness, van Cleve (1990) makes a very similar point and both authors link this emphasis on the nomological back to the British Emergentists.

The idea that emergence is a relation of merely nomological necessity delimits a certain group of emergent entities. This group does not match FundDep's Fundamental Dependents. To make this clear let us suppose, as has been argued by some, that the emergence relation is characterized by a causal relation ${ }^{14}$ and that this is nomologically but not metaphysically necessary. Returning to the case of conscious Nick and his unconscious body, BodyNick, let us also suppose that Nick and BodyNick are distinct and appropriately causally related: BodyNick causes or causally sustains Nick. Nick is then emergent from BodyNick on the view under consideration precisely because Nick is distinct from, but appropriately causally related to, BodyNick.

The fact that Nick is appropriately caused by BodyNick in the actual world does not entail that Nick will be appropriately caused by BodyNick in all possible worlds. There might be possible worlds where the causal laws are the same as in the actual world but where Nick is simply caused by something different from BodyNick. ${ }^{15}$ There might also be possible worlds where the causal laws are different from those in the actual world. ${ }^{16}$

Perhaps in an alternative possible world God has a necessary concrete body which appropriately causes Nick to exist [W2], or something non-concrete appropriately causes Nick [W3], or Nick is simply uncaused [W4]. One can speak of emergence in regard to these other possible worlds. If we take causation to be characteristic of emergence, then in W2 Nick emerges from God's body which

\footnotetext{
13 Cf. van Cleve (1990), McLaughlin (1997), Noordhof (2003), Chalmers (2006).

${ }^{14}$ Cf. O'Connor (2000: ch. 6), which takes consciousness to be emergent from, because causally grounded in, the properties of the body. Cf. also O'Connor and Wong (2005), and Lowe (2008).

${ }^{15}$ O'Connor (2000: 112) suggests that the causal grounding involved in a case of emergence needn't be the same in every possible world, because an emergent entity could exist in a different possible world without the causal grounds it has in the actual world. O'Connor and Wong (2005) also point out that it is coherent to suppose that the causal grounding could differ between worlds because causation itself differs.

${ }^{16}$ Cf. Sidelle (2002) and Beebee (2002), among others, for the idea that causal laws could differ between possible worlds.
} 
causes him, in W3 Nick emerges from something non-concrete which causes him, and in W4 Nick is not emergent at all (perhaps this is a world of disembodied spirits).

In short, if we adopt the current view that emergence is characterized by causation, then Nick is emergent from BodyNick in the actual world but is differently emergent or not emergent at all in other worlds, such as W2, W3, and W4. However, given FundDep's characterization of emergent entities as those that are Fundamental and Dependent, Nick will not be emergent in the actual world nor in any other possible world. The reason for this is that we can stipulate W2, W3, and W4 to all be worlds in which Nick exists but is not accompanied by any other concrete, contingent objects. So Nick is not a Dependent entity according to FundDep and therefore not an emergent entity.

The argument just given does not rely on taking the emergence relation to be characterized by causation. The argument only relies on emergence being a merely nomologically necessary relation and so all accounts of emergence incorporating this idea will diverge from FundDep in this way. I will not here assert the existence of W2, W3, or W4, or that something might be emergent in one world and not in another. Nevertheless, the point to note is that there is precedent to the idea that whether an entity is emergent can vary from world to world in a particular sort of way-something captured in the idea that cases of emergence are merely nomologically necessary. FundDep, identifying the emergent entities with Fundamental Dependent entities, does not reflect this variation. Therefore FundDep fails to accommodate what is arguably the most common understanding of emergence.

\section{Fundamental and Dependent but Not Emergent}

In this section I will argue that something might be Fundamental and Dependent but not emergent. If the emergent entities are the Fundamental Dependent entities, then any two concrete, contingent, Fundamental, entities which are necessarily covariant ${ }^{17}$ will be emergent. ${ }^{18}$ However, this seems incorrect. ${ }^{19}$ Things might be necessarily covariant and not emergent. For example, suppose that it were necessary that every time we have charge we have spin and vice versa, and that these were concrete, contingent, Fundamental properties. That wouldn't mean that charge would be an emergent property. Rather, neither charge nor spin appear to be good candidates for emergent properties. Neither entity appears to be sustained by or based in anything else.

Moreover, according to FundDep's characterization of the emergent entities as the Fundamental Dependent entities, a world in which there are only two necessarily

\footnotetext{
${ }^{17}$ By which I mean entities such that whenever one [or a counterpart of it] exists the other [or a counterpart of it] does too, and vice versa. Barnes's characterization of 'dependent on' mentioned in Sect. 5 appears to make space for necessary connections of just this sort. [Cf. Fine (1994) for arguments to show that metaphysically necessary covariance is not ontological dependence].

18 Assuming contingency and concreteness are essential properties of their bearers in this case.

19 Contra Barnes's consideration of the case of mass, shape, and size tropes, where Barnes seems to think that it is good that we are forced to say that such necessarily covariant properties are emergent.
} 
covariant, contingent, Fundamental, concrete entities will be a world in which everything is emergent. However, a world of only emergents appears to be impossible. Most prominent accounts of emergence take it to be an asymmetric relation. If this is correct, such a lone pair of necessarily covariant entities could not both be emergent, contra FundDep.

Historically this asymmetry has been present in views according to which emergent entities were composed of the entities that formed their bases, and this composition is asymmetric. For example, Broad (1925) required that for one entity to emerge from another the former must be at a higher ontological level than the latter, as determined by the asymmetric relation of composition. ${ }^{20}$ However, this asymmetry lingers in more recent views that do not require composition and even those that explicitly deny it. ${ }^{21}$ For example, Kim (1998: 11) argues that supervenience, common to many accounts of emergence, is asymmetric.

Suppose that we have a world containing just two necessarily covariant, contingent, concrete, Fundamental entities, $S$ and $C$. If we suppose that $S$ is emergent, then it must either be emergent from itself or from $\mathrm{C}$. However, if we follow the idea that emergence is asymmetric, then $\mathrm{S}$ cannot be emergent from itself. Therefore, if $\mathrm{S}$ is emergent, it must be emergent from C. Similar reasoning shows that if $\mathrm{C}$ is emergent, it must be emergent from $\mathrm{S}$, as it cannot be emergent from itself. It follows that if both $\mathrm{S}$ and $\mathrm{C}$ are emergent, then $\mathrm{S}$ must be emergent from $\mathrm{C}$ and $\mathrm{C}$ from $\mathrm{S}$, but this is a symmetric relationship. Therefore, given the relevant assumptions, it is not possible for both $\mathrm{S}$ and $\mathrm{C}$ to be emergent, contra FundDep's characterization of the situation.

FundDep thus appears to misclassify the emergent entities as the Fundamental Dependent entities, as there appear to be entities that are Fundamental and Dependent but not emergent. The point being made here is not that FundDep disagrees with some other characterizations of emergence and so must be wrong, but that FundDep does not match prominent working candidates and this ought to be noted. It might be that a thorough characterization of emergence does not exactly map onto original notions. However, a characterization that strays too far from these original notions might, for that reason, be thought to be capturing a different phenomenon.

\section{Moving Forward by Clarifying Dependence}

In the preceding sections I have argued that FundDep is not an adequate account of emergence. Perhaps this should not be a surprise, given that Barnes appears to admit as much by stating that she does not take what she says to provide definitive characterizations of Fundamentality or Dependence. However, this does leave two questions: firstly, how far has Barnes actually advanced our understanding of

\footnotetext{
${ }^{20}$ Cf. also Mill (1843).

21 Cf. Humphreys (1996) who denies an emergent entity must be composed out of its base, instead saying that emergence involves destruction of the base (arguably, however, there remains a relation of emergence here, insofar as we are able to say that one entity emerges from another).
} 
emergence if not via these characterizations, and secondly, how should we move forward from here?

It was the characterization of Dependence adopted in FundDep that led to it parting company with other characterizations of emergence as merely nomological and asymmetric. In order to improve upon FundDep, it thus appears one ought to improve upon its notion of Dependence, which I shall hereon refer to as Depl.

In a footnote, Barnes offers a second characterization of dependence, or specifically a characterization of the relation of dependence-on, Dep2:

$x$ is dependent on the $y$ s iff $x$ is dependent because it is part of its intrinsic nature that it bears relation $\mathrm{R}$ to things intrinsically like the $y \mathrm{~s}$. (2012: $881 \mathrm{ftnt}$.

16)

This might offer us some advance over Dep1. In Sect. 2, I argued FundDep was inadequate because it did not explicitly equip us with a way of distinguishing whether Nick was Dependent on BodyNick or BodyClare. Dep2 does offer us a possible solution to this in relation R: we might be able to say that Nick is R-related to BodyNick and not BodyClare, and hence dependent on and emergent from BodyNick but not BodyClare. However, Barnes deliberately leaves relation R uncharacterized, and so it is still not clear how to understand relation $\mathrm{R}$, nor how to apply it in practice (furthermore, if W2, W3, or W4 are possible worlds, Nick is not R-related to BodyNick).

Dep2 will also result in conflict with prominent accounts of emergence. Dep1 delimits a group of dependent entities. Dep2 identifies entities in this group as dependent on other specific things: those things they are R-related to. Therefore, in order to be Dependent according to Dep2 an entity must still be Dependent according to Dep1. Specifically, it will now satisfy Dep1 because it is a relatum of relation R. However, the argument of Sect. 3 showed that according to prominent accounts an entity may be considered emergent despite failing to qualify as Dependent according to Dep1 (because emergence is merely nomologically necessary, though an entity will be Dependent in every possible world if it is Dependent in any). We again have cases of emergent entities which are not Fundamental Dependent entities.

Secondly, returning to the argument of Sect. 4 above it was shown that Fundamental, contingent, concrete, necessarily covariant kinds of entities such as spin and charge may not be considered emergent on prominent accounts. However, such covariant kinds may qualify as Dependent according to Dep2. This is because the kind a fundamental entity belongs to is plausibly an intrinsic matter. Two fundamental entities that necessarily co-vary because of their kinds thus necessarily co-vary because of their intrinsic natures. Consequently, the relation of necessary covariance-or the relation between kinds that grounds it on this occasion-in this case fulfils two criteria: it stems from the intrinsic natures of the relata, and it ensures that the relata are Dependent by the standards of Dep1. We thus have a candidate for relation $\mathrm{R}$ in these cases, because the only criteria for relation $\mathrm{R}$ that Barnes makes explicit are these very same criteria: that it stems from the intrinsic nature of its relata, and that it ensures that those relata are Dependent by the standards of Dep1. These necessarily covariant entities would thus be characterized 
as Fundamental and Dependent, but they will not be characterized as emergent according to prominent characterisations of emergence. Further, Dep2 may also conflict with views that take the dependence at issue to be causal because causation is often thought to be an external relation and so not the intrinsic one given by R. ${ }^{22}$

Here we might distinguish two tasks: offering a general characterization of emergence that encompasses all existing accounts of emergence and offering a characterization of emergence fitting one common notion of emergence. If we are to attempt the latter task, we ought not to simply adopt FundDep. Further, we cannot simply rest with what Barnes has said, because it is important to be more specific about the relation of dependence: while her lack of commitment to her characterizations of this notion lets her sidestep seeming counterexamples, it also reveals a need for more detail and makes it questionable whether Barnes has advanced the debate. If common accounts of emergence are right, the dependence of emergent entities on their bases is not captured by Dep1 or Dep2; rather emergence is an asymmetric and merely nomological relation. I will not here detail or defend such a proposal, but the basic form might be given by:

$x$ is emergent from $y$ [or the $y$ s] if $x$ is Fundamental and $x$ is in relation $\mathrm{R}$ to $y$ [or the $y \mathrm{~s}$ ], where relation $\mathrm{R}$ is dependence, which is an asymmetrical and nomologically necessary but not metaphysically necessary relation.

This form requires more detail because it is plausible that not all merely nomological and asymmetric relations will qualify as dependence so intended. If one wished to be more specific than this they may add the notion of Fundamentality to a notion of dependence already mentioned in the literature, such as a form of supervenience or causation (considered as asymmetric and merely nomological). I leave detailed discussion of such views for elsewhere, but existent literature on these notions offers various suggestions for how the position might be considered. ${ }^{23}$

Alternatively, if we are interested in providing a general account of emergence we must look further than FundDep. This is true whether FundDep is characterized by Dep1 or Dep 2. A general account does not require that emergence is merely nomological, since some versions of emergence treat it as holding with metaphysical necessity (for example, O'Connor and Wong 2005). However, such an account should leave open the possibility that emergence might be merely nomological, which FundDep at present fails to do. Similarly, all versions of ontological emergence seem to agree that emergence is asymmetric, ${ }^{24}$ but this is not provided for by FundDep as it stands.

Which of these two tasks Barnes is most concerned with is not clear. However, she does express a concern with whether her characterization of emergence fits existent and common notions, ${ }^{25}$ and it is clear that it does not if we follow the characterizations of dependence that she offers. Conversely, if we do not follow Barnes's characterizations, accepting the caution she presents them with, then it is

\footnotetext{
22 This paper has greatly benefitted from the work of anonymous referees, one of whom made this point.

23 Cf, for example, van Cleve (1990), Chalmers (2006), and O'Connor and Wong (2005).

24 Asymmetry does seem to be generally accepted. Cf. O’Connor (1994, footnote 18).

25 Barnes (2012: p. 874 para. 4 and p. 886 para. 2).
} 
not clear how Barnes's account of emergence differs from, or hence advances, those already present in the literature.

\section{Conclusion}

Barnes has offered what she claims to be a new characterization of emergence: the emergent entities are the Dependent Fundamental entities. What makes this account new is not the expressions 'dependent' and 'fundamental', but Barnes's application of a particular metaontological interpretation of dependence and fundamentality. In this paper I have not questioned this metaontological stance, but the value of Barnes's application of it. I have argued that as she has characterized them, these notions do not provide an adequate account of emergence. This is primarily because an adequate account of dependence has not been provided: delimiting the relata of a kind of relation does not suffice to show where instances of that relation hold.

Furthermore, I have cast doubt over the adequacy of a characterization of emergence built on these notions of dependence and fundamentality by arguing that it excludes some entities commonly considered to be emergent and includes some entities commonly considered not to be emergent. Barnes's characterization of dependence fails to accommodate emergence that holds with merely nomological necessity and fails to ensure that emergence is asymmetric. I therefore conclude that in order to characterize emergence we need to go beyond what Barnes says, specifically concerning dependence.

Acknowledgements This paper has been written whilst working as a research fellow on the Durham Emergence Project, funded by The John Templeton Foundation.

Open Access This article is distributed under the terms of the Creative Commons Attribution 4.0 International License (http://creativecommons.org/licenses/by/4.0/), which permits unrestricted use, distribution, and reproduction in any medium, provided you give appropriate credit to the original author(s) and the source, provide a link to the Creative Commons license, and indicate if changes were made.

\section{References}

Barnes, E. (2012). Emergence and fundamentality. Mind, 121(484), 873-901.

Beebee, H. (2002). Contingent laws rule: Reply to bird. Analysis, 62(3), 252-255.

Broad, C. D. (1925). The mind and its place in nature. Cambridge: Keegan Paul.

Cameron, R. (2008). Truthmakers and ontological commitment. Philosophical Studies, 140, 1-18.

Chalmers, D. (2006). Strong and weak emergence. In P. Clayton \& P. Davies (Eds.), The re-emergence off emergence (pp. 244-255). Oxford: Oxford University Press.

Fine, K. (1994). Essence and modality. Philosophical Perspectives, 8, 1-16.

Humphreys, P. (1996). Aspects of emergence. Philosophical Topics, 24, 53-70.

Kim, J. (1998). Mind in a physical world. Cambridge: MIT Press.

Kim, J. (2006). Emergence: Core ideas and issues. Synthese, 151, 547-559.

Laughlin, R. B. (1999). Nobel lecture: Fractional quantization. Reviews of Modern Physics, 71, 863-874.

Lowe, E. J. (2008). Personal agency. Oxford: Oxford University Press.

Maudlin, T. (2007). The metaphysics within physics. Oxford: Oxford University Press.

McLaughlin, B. (1997). Emergence and supervenience. Intellectia, 25, 25-43. 
Mill, J. S. (1843/1919). A system of logic. London: Longmans.

Noordhof, P. (2003). Not old... But not that new either: Explicability, emergence and the characterisation of materialism. In S. Walter \& H.-D. Heckman (Eds.), Physicalism and mental causation: The metaphysics of mind and action (pp. 85-108). Charlottesville: Imprint Academic.

O’Connor, T. (1994). Emergent properties. American Philosophical Quarterly, 31(2), 91-104.

O’Connor, T. (2000). Persons and causes. Oxford: Oxford University Press.

O'Connor, T., \& Wong, H. Y. (2005). The metaphysics of emergence. Nous, 39, 658-678.

Rothschild, L. J. (2006). The role of emergence in biology. In P. Clayton \& P. Davies (Eds.), The reemergence off emergence (pp. 151-165). Oxford: Oxford University Press.

Sawyer, R. K. (2001). Emergence in sociology: Contemporary philosophy of mind and some implications for sociological theory. American Journal of Sociology, 107(3), 551-585.

Sidelle, A. (2002). On the metaphysical contingency of laws of nature. In J. Hawthorne \& T. Gendler (Eds.), Conceivability and possibility (pp. 309-336). Oxford: Oxford University Press.

van Cleve, J. (1990). Mind-dust or magic? Panpsychism versus emergence. Philosophical Perspectives, 4 , 215-226.

Van Gulick, R. (2001). Reduction, emergence and other recent options on the mind/body problem: A philosophic overview. Journal of Consciousness Studies, 8, 1-34.

Wilson, J. (1999). How superduper does a physicalist supervenience need to be? The Philosophical Quarterly, 49, 33-52.

Wilson, J. (2005). Supervenience-based formulations of physicalism. Nous, 39, 426-459.

Wilson, J. (2011). Non-reductive realization and the powers-based subset strategy. The Monist, 94, 121-154.

Wilson, J. (2013). Nonlinearity and metaphysical emergnce. In S. Mumford \& M. Tugby (Eds.), Metaphysics and science (pp. 189-226). Oxford: Oxford University Press.

Wilson, J. (2015). Metaphysical emergence: Weak or strong? In T. Bigaj \& C. Wüthrich (Eds.), Metaphysics in contemporary physics (pp. 251-306). Amsterdam/New York: Rodopi. 